TRANSACTIONS OF THE

AMERICAN MATHEMATICAL SOCIETY

Volume 351, Number 1, January 1999, Pages 109-130

S 0002-9947(99)02285-0

\title{
LARGE TIME ASYMPTOTICS OF SOLUTIONS TO THE GENERALIZED BENJAMIN-ONO EQUATION
}

\author{
NAKAO HAYASHI AND PAVEL I. NAUMKIN
}

\begin{abstract}
We study the asymptotic behavior for large time of solutions to the Cauchy problem for the generalized Benjamin-Ono (BO) equation: $u_{t}+$ $\left(|u|^{\rho-1} u\right)_{x}+\mathcal{H} u_{x x}=0$, where $\mathcal{H}$ is the Hilbert transform, $x, t \in \mathbf{R}$, when the initial data are small enough. If the power $\rho$ of the nonlinearity is greater than 3 , then the solution of the Cauchy problem has a quasilinear asymptotic behavior for large time. In the case $\rho=3$ critical for the asymptotic behavior i.e. for the modified Benjamin-Ono equation, we prove that the solutions have the same $L^{\infty}$ time decay as in the corresponding linear BO equation. Also we find the asymptotics for large time of the solutions of the Cauchy problem for the $\mathrm{BO}$ equation in the critical and noncritical cases. For the critical case, we prove the existence of modified scattering states if the initial function is sufficiently small in certain weighted Sobolev spaces. These modified scattering states differ from the free scattering states by a rapidly oscillating factor.
\end{abstract}

\section{INTRODUCTION}

In this paper we study the asymptotic behavior in time of solutions of the Cauchy problem for the generalized Benjamin-Ono (BO) equation

$$
\left\{\begin{array}{l}
u_{t}+\left(|u|^{\rho-1} u\right)_{x}+\mathcal{H} u_{x x}=0, \quad t, x \in \mathbf{R}, \\
u(0, x)=u_{0}(x), \quad x \in \mathbf{R} .
\end{array}\right.
$$

Here $\mathcal{H}=-\frac{1}{\pi} \int \frac{u(t, y)}{y-x} d y$ is the Hilbert transform and $u_{0}$ is a real valued function. We note that $\mathcal{H} \partial_{x}^{2}=\partial_{x}\left(-\partial_{x}^{2}\right)^{1 / 2}$.

The physical background of the Benjamin-Ono equation is discussed in $[5,25,32$, 34]. Exact solitary wave solutions were found in [4, 7], and the inverse scattering transform method was applied to the Cauchy problem (1.1) when $\rho=2$ in $[3,6$, $8,27]$. The Cauchy problem (1.1) has been intensively studied by many authors. The existence of solutions in the Sobolev space $H^{s}$ was proved in $[1,2,13,18,19$, $24,28,30,31]$ and the smoothing properties of solutions were studied in $[9,12,14$, $15,20,29,35]$. In the case $\rho \geq 5$ some estimates of the time decay of the solutions and the existence of the free evolution states were proved in the paper [21]. As far as we know there is no any other result on the asymptotic behavior of solutions of the Cauchy problem for the generalized BO equation (1.1). For small initial data in $H^{2,0} \cap H^{1,1}$, we prove that in the noncritical case $\rho>3$ the solutions of (1.1) are asymptotically free, i.e. tend to the solutions of the linear BO equation as $t \rightarrow \infty$. And for the critical case $\rho=3$ we prove that for small initial data in $H^{3,0} \cap H^{1,2}$

Received by the editors August 9, 1996.

1991 Mathematics Subject Classification. Primary 35Q55.

(C)1999 American Mathematical Society 
the solutions decay as $t \rightarrow \infty$ in $L^{\infty}$ norm at the same rate $t^{-\frac{1}{2}}$ as the linear BO equation. Also we construct the modified scattering states and prove the existence of the inverse modified wave operators. We note that the modified wave operators and the inverse modified wave operators for the nonlinear Schrödinger equations were constructed previously in papers $[11,17,26]$ and $[16,22,23]$, respectively.

Before stating our results we give

Notation and function spaces. Let $\mathcal{F} \phi$ or $\hat{\phi}$ be the Fourier transform of $\phi$, defined by

$$
\mathcal{F} \phi(\xi)=\frac{1}{\sqrt{2 \pi}} \int e^{-i x \xi} \phi(x) d x
$$

The inverse Fourier transform $\mathcal{F}^{-1}$ is given by

$$
\mathcal{F}^{-1} \phi(x)=\frac{1}{\sqrt{2 \pi}} \int e^{i x \xi} \phi(\xi) d \xi .
$$

Let $U(t)$ be the free Benjamin-Ono evolution group defined by

$$
\begin{aligned}
U(t) \phi & =\mathcal{F}^{-1} e^{-i t \xi|\xi|} \hat{\phi}(\xi) \\
& =\frac{1}{2 \pi} \int d y \phi(y) \int d \xi e^{i \xi(x-y)-i t \xi|\xi|} \\
& =\frac{1}{\sqrt{|t|}} \operatorname{Re} \int e^{\frac{i(x-y)^{2}}{4|t|}} E\left(\frac{(y-x) \sqrt{|t|}}{2 t}\right) \phi(y) d y .
\end{aligned}
$$

After the change of variables $\xi-\frac{\eta}{2 t}=\frac{z}{\sqrt{|t|}}$ we obtain

$$
\begin{aligned}
& \frac{1}{2 \pi} \int e^{i \xi \eta-i t \xi|\xi|} d \xi=\frac{1}{\pi} \operatorname{Re} \int_{0}^{\infty} e^{i \xi \eta-i t \xi^{2}} d \xi \\
& =\frac{1}{\pi \sqrt{|t|}} \operatorname{Re} e^{\frac{i \eta^{2}}{4|t|}} \int_{\frac{-\eta \sqrt{|t|}}{2 t}}^{\infty} e^{-i z^{2}} d z=\frac{1}{\sqrt{|t|}} \operatorname{Re} e^{\frac{i \eta^{2}}{4|t|}} E\left(\frac{-\eta \sqrt{|t|}}{2 t}\right),
\end{aligned}
$$

where

$$
E(\eta)=\frac{1}{\pi} \int_{\eta}^{\infty} e^{-i z^{2}} d z
$$

We introduce some function spaces. $L^{p}=\left\{\phi \in \mathcal{S}^{\prime} ;\|\phi\|_{p}<\infty\right\}$, where $\|\phi\|_{p}=$ $\left(\int|\phi(x)|^{p} d x\right)^{1 / p}$ if $1 \leq p<\infty$ and $\|\phi\|_{\infty}=\operatorname{ess} \sup \{|\phi(x)| ; x \in \mathbf{R}\}$ if $p=\infty$. For simplicity we let $\|\phi\|=\|\phi\|_{2}$. The weighted Sobolev space $H_{p}^{m, s}$ is defined by $H_{p}^{m, s}=\left\{\phi \in \mathcal{S}^{\prime} ;\|\phi\|_{m, s, p}=\left\|\left(1+|x|^{2}\right)^{s / 2}\left(1-\partial_{x}^{2}\right)^{m / 2} \phi\right\|_{p}<\infty\right\}, m, s \in \mathbf{R}$, $1 \leq p \leq \infty$; for short we put $H^{m, s}=H_{2}^{m, s},\|\cdot\|_{m, s}=\|\cdot\|_{m, s, 2}$. We let $(\psi, \phi)=$ $\int \psi \cdot \bar{\phi} d x$ and let $C(I ; H)$ be the space of continuous functions from an interval $I$ to a Banach space $H$. Different positive constants may be denoted by the same letter $C$. If necessary, by $C(*, \cdots, *)$ we denote constants depending on the quantities appearing in the parentheses.

We now state our results.

Theorem 1.1. Assume that the initial data $u_{0}$ is real, with $u_{0} \in H^{2,0} \cap H^{1,1}$ and $\left\|u_{0}\right\|_{2,0}+\left\|u_{0}\right\|_{1,1}=\epsilon$, where $\epsilon$ is sufficiently small. Then there exists a unique global solution $u$ of the Cauchy problem (1.1) with $\rho>3$ such that

$$
u \in C\left(\mathbf{R} ; H^{2,0} \cap H^{1,1}\right),
$$




$$
\|u(t)\|_{\infty}+\left\|u_{x}(t)\right\|_{\infty} \leq C \epsilon(1+|t|)^{-1 / 2} .
$$

Theorem 1.2. Let $u$ be the solution of (1.1) with $\rho>3$ obtained in Theorem 1.1. Then for any $u_{0} \in H^{2,0} \cap H^{1,1}$ there exists a unique function $V \in H^{1,0} \cap H^{0,1}$ such that

$$
\left\|U(-t) u(t)-\mathcal{F}^{-1} V\right\|_{1,0}+\left\|U(-t) u(t)-\mathcal{F}^{-1} V\right\|_{0,1} \leq C \epsilon t^{-\frac{1}{2} \min \{1, \rho-3\}} \quad \text { for } t \geq 1 .
$$

Furthermore, for large time $t$ we have the asymptotic formula

$$
u(t, x)=\sqrt{\frac{2 \pi}{t}} \operatorname{Re}\left(E\left(-\frac{x}{2 \sqrt{t}}\right) V\left(\frac{x}{2 t}\right) \exp \left(i \frac{x^{2}}{4 t}\right)\right)+O\left(\epsilon t^{-\frac{1}{2}-\frac{1}{2} \min \{1, \rho-3\}}\right) .
$$

Theorem 1.3. Assume that the initial data $u_{0}$ is real, with $u_{0} \in H^{3,0} \cap H^{1,2}$ and $\left\|u_{0}\right\|_{3,0}+\left\|u_{0}\right\|_{1,2}=\epsilon$, where $\epsilon$ is sufficiently small. Then there exists a unique global solution $u$ of the Cauchy problem for the modified BO equation (1.1) with $\rho=3$ such that

$$
\begin{gathered}
u \in C\left(\mathbf{R} ; H^{3,0} \cap H^{1,2}\right), \\
\|u(t)\|_{\infty}+\left\|u_{x}(t)\right\|_{\infty} \leq C \epsilon(1+|t|)^{-1 / 2} .
\end{gathered}
$$

Theorem 1.4. Let $u$ be the solution of the Cauchy problem (1.1) with $\rho=3$ obtained in Theorem 1.3. Then for any $u_{0} \in H^{3,0} \cap H^{1,2}$ there exist unique functions $W$ and $\Phi \in L^{\infty}$ such that

$$
\begin{gathered}
\left.\| \mathcal{F}(U(-t) u)(t) \exp \left(3 i \pi p \int_{1}^{t}|\hat{u}(\tau)|^{2}\right) \frac{d \tau}{\tau}\right)-W \|_{\infty} \leq C \epsilon t^{-\alpha} \quad \text { for } \quad t \geq 1, \\
\left\|\pi p \int_{1}^{t}|\hat{u}(\tau)|^{2} \frac{d \tau}{\tau}-\pi p|W|^{2} \log t-\Phi\right\|_{\infty} \leq C \epsilon t^{-\alpha} \quad \text { for } \quad t \geq 1,
\end{gathered}
$$

where $0<\alpha<1 / 4-C \epsilon$ and $\Phi$ is a real valued function. Furthermore, for large time $t$ we have the asymptotic formula

$$
\begin{aligned}
& u(t, x)=\sqrt{\frac{2 \pi}{t}} \operatorname{Re}\left(E\left(-\frac{x}{2 \sqrt{t}}\right) W\left(\frac{x}{2 t}\right) \exp \left(i \frac{|x|^{2}}{4 t}-3 i \frac{x}{2 t} \pi\left|W\left(\frac{x}{2 t}\right)\right|^{2} \log t-3 i \Phi\left(\frac{x}{2 t}\right)\right)\right. \\
&(1.7) \quad+O\left(\epsilon t^{-1 / 2-\alpha}\right),
\end{aligned}
$$

and for $t \geq 1$

$$
\left\|\mathcal{F}(U(-t) u)(t)-W \exp \left(-i 3 \pi p|W|^{2} \log t-3 i \Phi\right)\right\|_{\infty} \leq C \epsilon t^{-\alpha}
$$

In what follows, for simplicity we only consider positive time $t$.

Remark 1.1. The inequalities (1.2), (1.5), (1.6) and (1.8) show that the functions $V, W$ and $\Phi$ can be calculated approximately via the initial data $u_{0}$, and (1.2) means the existence of the scattering states in the noncritical case $\rho>3$.

Remark 1.2. Our method can also be applied to the equation

$$
\left\{\begin{array}{l}
u_{t}+(f(u))_{x}+\mathcal{H} u_{x x}=0, \quad t, x \in \mathbf{R}, \\
u(0, x)=u_{0}(x), \quad x \in \mathbf{R},
\end{array}\right.
$$

where $f(u)=O\left(|u|^{\rho-1} u\right)$ for small $u$, where $\rho \geq 3$. 
We organize our paper as follows. In Section 2 we give some preliminary estimates. The Sobolev inequality is stated in Lemma 2.1. Lemma 2.2 says that the time decay of the function can be represented by the free evolution group of the Benjamin-Ono operator. Lemmas 2.3 and 2.4 are used in the proof of Lemma 2.5, and Lemma 2.5 is necessary for the proof of Lemma 4.2 to treat the nonlinear term in the critical case $\rho=3$. In Section 3 we prove Theorems 1.1 and 1.2 , and in Section 4 we prove Theorems 1.3 and 1.4 by a priori estimates of local solutions to (1.1) established in Lemma 3.1 and Lemmas 4.1-4.2 respectively. Our approach for the critical case $\rho=3$ consists of two parts. First, in Lemma 4.1 we establish some rough a priori estimates of the solution in the norms $\|u\|_{3,0}$ and $\|x U(-t) u\|_{2,0}$, assuming that the precise decay estimate of the solution $\|u\|_{\infty}+\left\|u_{x}\right\|_{\infty} \leq C(1+|t|)^{-1 / 2}$ is already known. In order to obtain the desired estimates, we apply the dilation operator $I=x+2 t \partial_{t} \int_{-\infty}^{x} d x^{\prime}$, which was previously used in [15] to obtain the smoothing properties of the BO equation. Then in Lemma 4.2 we prove the precise decay estimate of the solution, $\|u\|_{\infty}+\left\|u_{x}\right\|_{\infty} \leq C(1+|t|)^{-1 / 2}$, assuming that the rough estimates of the solution in the norm $\|u\|_{3,0}+\|x U(-t) u\|_{2,0}$ are fulfilled.

\section{Preliminaries}

Lemma 2.1. Let $q, r$ be any numbers satisfying $1 \leq q, r \leq \infty$, and let $j, m$ be any numbers satisfying $0 \leq j<m$. If $u \in H_{r}^{m, 0} \cap L^{q}$, then

$$
\left\|\left(-\partial_{x}^{2}\right)^{j / 2} u\right\|_{p} \leq C\left\|\left(-\partial_{x}^{2}\right)^{m / 2} u\right\|_{r}^{a}\|u\|_{q}^{1-a},
$$

where $1 / p=j+a((1 / r)-m)+(1-a) / q$ for all a in the interval $j / m \leq a \leq 1$, where $C$ is a constant depending only on $m, j, q, r, a$, with the following exception: if $m-j-1 / r$ is a nonnegative integer, then the above inequality holds for any $j / m \leq a<1$.

For Lemma 2.1 see, e.g., [10, 33].

Lemma 2.2. Let $u(t, x)$ be a smooth function. Then, for all $t \geq 1$,

$$
\begin{gathered}
\|u(t)\|_{\infty} \leq t^{-1 / 2}\|U(-t) u(t)\|_{0,1}, \\
\|u(t)\|_{\infty} \leq C t^{-1 / 2}\|\mathcal{F} u(t)\|_{\infty}+C t^{-1 / 2-\nu}\|U(-t) u(t)\|_{0,1},
\end{gathered}
$$

where $\nu \in[0,1 / 2)$ and $U(t)$ is the free Benjamin-Ono evolution group.

Proof. We have the identity

$$
u(t, x)=U(t) U(-t) u(t, x)=\frac{1}{\sqrt{t}} \operatorname{Re}\left\{\int e^{i(x-y)^{2} / 4 t} E\left(\frac{y-x}{2 \sqrt{t}}\right) U(-t) u(t, y) d y\right\},
$$

where $E(x)=\frac{1}{\pi} \int_{x}^{\infty} e^{-i y^{2}} d y$. Let us consider only the case $t \geq 1$. The first estimate (2.1) easily follows from (2.3). To obtain (2.2) we write (2.3) in the following way:

$$
\begin{aligned}
u(t, x) & =\operatorname{Re}\left\{\frac{e^{i x^{2} / 4 t}}{\sqrt{t}} \int e^{\frac{-i x y}{2 t}} U(-t) u(t, y) e^{i y^{2} / 4 t} E\left(\frac{y-x}{2 \sqrt{t}}\right) d y\right\} \\
& =\sqrt{\frac{2 \pi}{t}} \operatorname{Re}\left\{e^{i x^{2} / 4 t} E\left(-\frac{x}{2 \sqrt{t}}\right)(\mathcal{F} U(-t) u(t))\left(t, \frac{x}{2 t}\right)\right\}+\mathcal{R}(t, x),
\end{aligned}
$$


where

$$
\begin{aligned}
\mathcal{R}(t, x)=\sqrt{\frac{2 \pi}{t}} \operatorname{Re}\left\{e^{i x^{2} / 4 t}\right. & \int e^{-i x y / 2 t}\left(e^{i y^{2} / 4 t} E\left(\frac{y-x}{2 \sqrt{t}}\right)\right. \\
& \left.\left.-E\left(-\frac{x}{2 \sqrt{t}}\right)\right) U(-t) u(t, y) d y\right\} .
\end{aligned}
$$

We let $\nu$ satisfy $0 \leq \nu<1 / 2$. Then we have the estimate

$$
\left|e^{i y^{2} / 4 t}-1\right|=2\left|\sin \frac{y^{2}}{4 t}\right| \leq C \frac{|y|^{\nu}}{|t|^{\nu / 2}}
$$

and by the Lagrange formula we get

$$
\left|E\left(\frac{y-x}{2 \sqrt{t}}\right)-E\left(-\frac{x}{2 \sqrt{t}}\right)\right| \leq C\left|E^{\prime}(\kappa) \frac{y}{\sqrt{t}}\right|^{\nu} \leq C \frac{|y|^{\nu}}{t^{\nu / 2}},
$$

where $\kappa$ is an intermediate point. Thus by the Schwarz inequality we have

$$
\|\mathcal{R}\|_{\infty} \leq C t^{-1 / 2-\nu / 2}\left\||y|^{\nu} U(-t) u(t, y)\right\|_{1} \leq C t^{-1 / 2-\nu / 2}\|U(-t) u(t)\|_{0,1} .
$$

From (2.4) and (2.5) we obtain (2.2). Lemma 2.2 is proved.

In the next lemma we estimate the following integral:

$$
\Omega_{1}=\max (1, \eta) \int_{\eta}^{\infty} e^{i x^{2}} \bar{E}(a x+\chi) d x
$$

where $\eta, a, \chi \in \mathbf{R}, \quad \bar{E}(x)=\frac{1}{\pi} \int_{x}^{\infty} e^{i y^{2}} d y$.

Lemma 2.3. The estimate

$$
\sup _{\chi, \eta \in \mathbf{R}}\left|\Omega_{1}\right| \leq C
$$

is valid, where $C=C(a)$.

Proof. Using the identity

$$
e^{i x^{2}}=\frac{1}{1+2 i x^{2}} \frac{d}{d x}\left(x e^{i x^{2}}\right),
$$

we integrate by parts with respect to $x$ to get

$$
\begin{aligned}
\Omega_{1}=\max (1, \eta)\{ & -\frac{\eta e^{i \eta^{2}}}{1+2 i \eta^{2}} \bar{E}(a \eta+\chi) \\
& \left.+\int_{\eta}^{\infty} \frac{4 i x^{2} e^{i x^{2}}}{\left(1+2 i x^{2}\right)^{2}} \bar{E}(a x+\chi) d x+\int_{\eta}^{\infty} \frac{a x e^{i x^{2}+i(a x+\chi)^{2}} d x}{\pi\left(1+2 i x^{2}\right)}\right\}
\end{aligned}
$$

Hence we obtain

$$
\left|\Omega_{1}\right| \leq C \max (1, \eta)\left\{\frac{1}{1+|\eta|}+\int_{\eta}^{\infty} \frac{d x}{1+x^{2}}+\left|\int_{\eta}^{\infty} e^{i x^{2}\left(1+a^{2}\right)+2 i a \chi x} \frac{x d x}{1+2 i x^{2}}\right|\right\} .
$$


Now consider the case $\eta \geq 1$. Using the Bonnet theorem on the mean value of the conditionally convergent integral, we get

$$
\begin{aligned}
& \left|\int_{\eta}^{\infty} e^{i x^{2}\left(1+a^{2}\right)+2 i a \chi x} \frac{x d x}{1+2 i x^{2}}\right| \\
& \quad=\left|\int_{\eta}^{\infty} e^{i x^{2}\left(1+a^{2}\right)+2 i a \chi x}\left(\frac{1}{2 i x\left(1+2 i x^{2}\right)}-\frac{1}{2 i x}\right) d x\right| \\
& \quad \leq C \int_{\eta}^{\infty} \frac{d x}{x^{3}}+C\left|\int_{\eta}^{\infty} e^{i x^{2}\left(1+a^{2}\right)+2 i a \chi x} \frac{d x}{x}\right| \\
& \quad \leq \frac{C}{\eta^{2}}+\frac{C}{\eta}\left|\int_{\eta}^{\kappa} e^{i x^{2}\left(1+a^{2}\right)+2 i a \chi x} d x\right| \leq \frac{C}{\eta},
\end{aligned}
$$

where $\kappa \in(\eta, \infty)$ is an intermediate point. Thus we have $\left|\Omega_{1}\right| \leq C$. The case $\eta<1$ can be considered analogously. Lemma 2.3 is proved.

Let us now estimate the following integral:

$$
\Omega_{2}=P^{1-\nu} t^{-\nu / 2}(\max (1, \min (\eta,|\chi|)))^{\nu} \int_{\eta}^{\infty} \frac{e^{i x^{2}} E(\sigma x+\chi) d x}{P+(x-\eta) / \sqrt{t}},
$$

where $P, t \geq 1, \eta, \chi \in \mathbf{R}, \nu \in(0,1), \sigma=+1$ or -1 .

Lemma 2.4. We have the estimate

$$
\sup _{P, t \geq 1, \chi, \eta \in \mathbf{R}}\left|\Omega_{2}\right| \leq C,
$$

where $C=C(\gamma)$.

Proof. Using (2.6), we integrate by parts with respect to $x$, and get

$$
\begin{aligned}
\Omega_{2} & =-P^{1-\nu} t^{-\nu / 2}\left(\operatorname { m a x } ( 1 , \operatorname { m i n } ( \eta , \chi ) ) ^ { \nu } \left\{\frac{\eta E(\sigma \eta+\chi) e^{i \eta^{2}}}{P\left(1+2 i \eta^{2}\right)}\right.\right. \\
& -\int_{\eta}^{\infty}\left(\frac{4 i x^{2}}{1+2 i x^{2}}+\frac{x}{P \sqrt{t}+x-\eta}\right) \frac{E(\sigma x+\chi) e^{i x^{2}} d x}{(P+(x-\eta) / \sqrt{t})\left(1+2 i x^{2}\right)} \\
& \left.-\frac{\sigma}{\pi} \int_{\eta}^{\infty} \frac{e^{-2 i \sigma \chi x-i \chi^{2}} x d x}{(P+(x-\eta) / \sqrt{t})\left(1+2 i x^{2}\right)}\right\} .
\end{aligned}
$$

Hence

$$
\begin{aligned}
\left|\Omega_{2}\right| & \leq C+C \max (1, \eta) \int_{\eta}^{\infty} \frac{d x}{1+x^{2}} \\
& +C(\max (1, \eta))^{\nu} \int_{\eta}^{\infty} \frac{d x}{(1+|x|)(P \sqrt{t}+x-\eta)}+\Omega_{3} \leq C+\Omega_{3},
\end{aligned}
$$

where

$$
\Omega_{3}=P^{1-\nu} t^{-\nu / 2}(\max (1, \min (\eta, \chi)))^{\nu}\left|\int_{\eta}^{\infty} \frac{e^{-2 i \sigma x \chi} x d x}{(P+(x-\eta) / \sqrt{t})\left(1+2 i x^{2}\right)}\right| .
$$

If $|\chi| \leq 1$ we estimate the last integral $\Omega_{3}$ in the following manner:

$$
\begin{aligned}
\left|\Omega_{3}\right| & \leq C t^{-\nu / 2} \int_{\eta}^{\infty} \frac{d x}{(1+|x|)(P+(x-\eta) / \sqrt{t})^{\nu}} \leq C \int_{\eta}^{\infty} \frac{d x}{(1+|x|)(1+x-\eta)^{\nu}} \\
& \leq C \int_{\eta}^{\infty}\left((1+|x|)^{-1-\nu}+(1+x-\eta)^{-1-\nu}\right) d x \leq C .
\end{aligned}
$$


And if $|\chi| \geq 1$, we integrate one more time by parts with respect to $x$, and get

$$
\begin{aligned}
\Omega_{3} \leq & C P^{1-\nu} t^{-\nu / 2}|\chi|^{\nu} \mid \frac{\eta e^{-2 i \sigma \eta \chi}}{2 i \chi P\left(1+2 i \eta^{2}\right)} \\
& -\int_{\eta}^{\infty}\left(\frac{2 i x^{2}-1}{1+2 i x^{2}}+\frac{x}{P \sqrt{t}+x-\eta}\right) \frac{e^{-2 i \sigma x \chi} d x}{2 i \chi\left(1+2 i x^{2}\right)(P+(x-\eta) / \sqrt{t})} \mid \\
\leq & C+C \int_{\eta}^{\infty}\left(\frac{1}{1+x^{2}}+\frac{1}{(1+|x|)(1+x-\eta)}\right) d x \leq C .
\end{aligned}
$$

From (2.7) and (2.8) we obtain the result. Lemma 2.4 is proved.

In the next lemma we consider the following integral:

$$
\mathcal{N}(t, p)=(i p)^{k+1} \iint e^{i t \mathcal{L}} \hat{v}\left(t, \xi_{1}\right) \hat{v}\left(t, \xi_{2}\right) \hat{v}\left(t, \xi_{3}\right) d \xi_{1} d \xi_{2},
$$

where $\mathcal{L}=-p^{2}+\xi_{1}\left|\xi_{1}\right|+\xi_{2}\left|\xi_{2}\right|+\xi_{3}\left|\xi_{3}\right|, \quad \xi_{3}=p-\xi_{1}-\xi_{2}, \quad k=0,1, \quad t, p>0$.

Lemma 2.5. Let $v$ be a real function, $v \in H^{2,1}$ and $t, p>0$. Then the representation

$$
\mathcal{N}(t, p)=\frac{i \pi(i p)^{k+1}}{t \sqrt{3}} e^{-2 i t p^{2} / 3} \hat{v}^{3}\left(t, \frac{p}{3}\right)+\frac{3 \pi(i p)^{k+1}}{t}|\hat{v}(t, p)|^{2} \hat{v}(t, p)+\mathcal{R}(t, p)
$$

is valid with the estimate

$$
\|\mathcal{R}\|_{\infty} \leq C t^{-1-\gamma}\|v\|_{2,1}^{3},
$$

where $\gamma \in(0,1 / 4), \quad k=0,1$.

Proof. Since the function $e^{i t \mathcal{L}} \hat{v}\left(t, \xi_{1}\right) \hat{v}\left(t, \xi_{2}\right) \hat{v}\left(t, \xi_{3}\right)$, where $\mathcal{L}=-p^{2}+\xi_{1}\left|\xi_{1}\right|+\xi_{2}\left|\xi_{2}\right|+$ $\xi_{3}\left|\xi_{3}\right|, \quad \xi_{3}=p-\xi_{1}-\xi_{2}$, is symmetric with respect to the variables $\xi_{1}, \xi_{2}, \xi_{3}$, we can write $\mathcal{N}$ as a sum of the following three integrals:

$$
\mathcal{N}=Q_{1}+Q_{2}+Q_{3},
$$

where

$$
\begin{aligned}
& Q_{1}=(i p)^{k+1} \iint_{\Lambda_{1}} e^{i t \mathcal{L}_{1}} \hat{v}\left(t, \xi_{1}\right) \hat{v}\left(t, \xi_{2}\right) \hat{v}\left(t, \xi_{3}\right) d \xi_{1} d \xi_{2}, \\
& Q_{j}=3(i p)^{k+1} \iint_{\Lambda_{j}} e^{i t \mathcal{L}_{j}} \hat{v}\left(t, \xi_{1}\right) \hat{v}\left(t, \xi_{2}\right) \hat{v}\left(t, \xi_{3}\right) d \xi_{1} d \xi_{2}, \quad j=2,3, \\
& \Lambda_{1}=\left\{\left(\xi_{1}, \xi_{2}\right) \in \mathbf{R}^{2}: \xi_{1}>0, \xi_{2}>0, \xi_{3}>0\right\}, \quad \mathcal{L}_{1}=-p^{2}+\xi_{1}^{2}+\xi_{2}^{2}+\xi_{3}^{2}, \\
& \Lambda_{2}=\left\{\left(\xi_{1}, \xi_{2}\right) \in \mathbf{R}^{2}: \xi_{1}<0, \xi_{2}<0, \xi_{3}>0\right\}, \quad \mathcal{L}_{2}=-p^{2}-\xi_{1}^{2}-\xi_{2}^{2}+\xi_{3}^{2}, \\
& \Lambda_{3}=\left\{\left(\xi_{1}, \xi_{2}\right) \in \mathbf{R}^{2}: \xi_{1}>0, \xi_{2}>0, \xi_{3}<0\right\}, \quad \mathcal{L}_{3}=-p^{2}+\xi_{1}^{2}+\xi_{2}^{2}-\xi_{3}^{2} .
\end{aligned}
$$

In the first integral $Q_{1}$ we make the change of variables $\xi_{1}=p / 3+s / \sqrt{3}-q, \quad \xi_{2}=$ $p / 3+s / \sqrt{3}+q$. Then $\xi_{3}=p / 3-2 s / \sqrt{3}$, and thus we obtain

$$
Q_{1}=\frac{2(i p)^{k+1}}{\sqrt{3}} \int_{-p / \sqrt{3}}^{p / 2 \sqrt{3}} d s \int_{-p / 3-s / \sqrt{3}}^{p / 3+s / \sqrt{3}} d q e^{i t \overline{\mathcal{L}}_{1}} \hat{v}\left(t, \xi_{1}\right) \hat{v}\left(t, \xi_{2}\right) \hat{v}\left(t, \xi_{3}\right) .
$$


And in the integrals $Q_{2}$ and $Q_{3}$ we make the change of variables $\xi_{1}=p / 3+r-$ $q, \quad \xi_{2}=p / 3+r+q$. Then $\xi_{3}=p / 3-2 r$, and we obtain

$$
\begin{aligned}
Q_{2}=6 i p \int_{-\infty}^{-p / 3} d r \int_{p / 3+r}^{-p / 3-r} d q e^{i t \overline{\mathcal{L}}_{2}} \hat{v}\left(t, \xi_{1}\right) \hat{v}\left(t, \xi_{2}\right) \hat{v}\left(t, \xi_{3}\right) d \xi_{1} d \xi_{2} \\
=6 i p \int_{-\infty}^{-p / 3} d r \int_{p / 3+r}^{-p / 3-r} d q e^{i t \overline{\mathcal{L}}_{2}}\left(i \xi_{1}+i \xi_{2}+i \xi_{3}\right)^{k} \\
\\
\times \frac{1+\xi_{3}}{1+\xi_{3}} \hat{v}\left(t, \xi_{1}\right) \hat{v}\left(t, \xi_{2}\right) \hat{v}\left(t, \xi_{3}\right) d \xi_{1} d \xi_{2}
\end{aligned}
$$

and

$$
Q_{3}=6(i p)^{k+1} \int_{p / 6}^{\infty} d r \int_{-p / 3-r}^{p / 3+r} d q e^{\overline{\mathcal{L}}_{3}} \hat{v}\left(t, \xi_{1}\right) \hat{v}\left(t, \xi_{2}\right) \hat{v}\left(t, \xi_{3}\right)
$$

where $\overline{\mathcal{L}}_{1}=-2 p^{2} / 3+2 s^{2}+2 q^{2}, \quad \overline{\mathcal{L}}_{2}=-2 p^{2}+2(r-2 p / 3)^{2}-2 q^{2}$, and $\overline{\mathcal{L}}_{3}=$ $-2(r-2 p / 3)^{2}+2 q^{2}$. Note that the integral $Q_{1}$ is symmetric with respect to the turning of the coordinate axis to the angle $2 \pi / 3$; therefore we can write the representation

$$
\begin{gathered}
\int_{-p / \sqrt{3}}^{p / 2 \sqrt{3}} d s \int_{-p / 3-s / \sqrt{3}}^{p / 3+s / \sqrt{3}} d q=3 \int_{0}^{p / 2 \sqrt{3}} d s \int_{-s \sqrt{3}}^{s \sqrt{3}} d q \\
=3 \int_{0}^{\infty} d s \int_{-s \sqrt{3}}^{s \sqrt{3}} d q-3 \int_{p / 2 \sqrt{3}}^{\infty} d s \int_{-s \sqrt{3}}^{s \sqrt{3}} d q \\
=\iint d s d q-3 \int_{p / 2 \sqrt{3}}^{\infty} d s \int_{-s \sqrt{3}}^{s \sqrt{3}} d q,
\end{gathered}
$$

and so

$$
\begin{aligned}
Q_{1}=\frac{2 e^{-2 i t p^{2} / 3}(i p)^{k+1}}{\sqrt{3}(1+i p)^{k+1}}\left(\iint\right. & \left.d s d q-3 \int_{p / 2 \sqrt{3}}^{\infty} d s \int_{-s \sqrt{3}}^{s \sqrt{3}} d q\right) e^{2 i t s^{2}+2 i t q^{2}} \\
& \times\left(1+i \xi_{1}+i \xi_{2}+i \xi_{3}\right)^{k+1} \hat{v}\left(t, \xi_{1}\right) \hat{v}\left(t, \xi_{2}\right) \hat{v}\left(t, \xi_{3}\right) .
\end{aligned}
$$

We now represent the domain of integration in the integral $Q_{3}$ in the following manner:

$$
\begin{aligned}
\int_{p / 6}^{\infty} d r \int_{-p / 3-r}^{p / 3+r} d q & =\iint d r d q-\int_{-\infty}^{p / 6} d r \int d q \\
& -\int_{p / 6}^{\infty} d r \int_{p / 3+r}^{\infty} d q-\int_{p / 6}^{\infty} d r \int_{-\infty}^{-p / 3-r} d q
\end{aligned}
$$


The last two integrals in (2.13) coincide in view of the symmetry with respect to $\xi_{1}$ and $\xi_{2}$. Thus we get

$$
\begin{aligned}
& Q_{3}=6 \iint d r d q e^{i t \overline{\mathcal{L}}_{3}}\left(i \xi_{1}+i \xi_{2}+i \xi_{3}\right)^{k+1} \hat{v}\left(t, \xi_{1}\right) \hat{v}\left(t, \xi_{2}\right) \hat{v}\left(t, \xi_{3}\right) \\
& -\frac{6 i p}{1+i p} \int_{-\infty}^{p / 6} d r \int d q e^{i t \overline{\mathcal{L}}_{3}}\left(i \xi_{1}+i \xi_{2}+i \xi_{3}\right)^{k} \\
& \quad \times\left(1+i \xi_{1}+i \xi_{2}+i \xi_{3}\right) \hat{v}\left(t, \xi_{1}\right) \hat{v}\left(t, \xi_{2}\right) \hat{v}\left(t, \xi_{3}\right) \\
& \quad-12 i p \int_{p / 6}^{\infty} d r \int_{p / 3+r}^{\infty} d q e^{i t \overline{\mathcal{L}}_{3}}\left(i \xi_{1}+i \xi_{2}+i \xi_{3}\right)^{k} \frac{1+\xi_{2}+\xi_{3} / 2}{1+\xi_{2}+\xi_{3} / 2} \hat{v}\left(t, \xi_{1}\right) \hat{v}\left(t, \xi_{2}\right) \hat{v}\left(t, \xi_{3}\right) .
\end{aligned}
$$

Now we substitute $\hat{v}=\mathcal{F} v$; then, using the identity

$$
\int e^{i t(r-a)^{2}} d r=\sqrt{\frac{\pi}{t}} e^{i \pi / 4}
$$

after simple calculations we get

$$
\begin{aligned}
Q_{1}= & \frac{2 e^{-2 i t p^{2} / 3}}{\sqrt{3}(2 \pi)^{3 / 2}}\left(\frac{i p}{1+i p}\right)^{k+1} \\
& \times \iiint \exp \left(-i p(x+y+z) / 3-i\left(x^{2}+y^{2}+z^{2}-x y-x z-y z\right) / 6 t\right) \\
& \times\left(\int d s \int d q-3 \int_{p / 2 \sqrt{3}}^{\infty} d s \int_{-s \sqrt{3}}^{s \sqrt{3}} d q\right) e^{2 i t(s-\lambda / \sqrt{3})^{2}+2 i t(q-\mu)^{2}} \\
& \times\left(1+\partial_{x}+\partial_{y}+\partial_{z}\right)^{k+1} v(t, x) v(t, y) v(t, z) d x d y d z \\
= & \frac{i \pi(i p)^{k+1} e^{-2 i t p^{2} / 3}}{t \sqrt{3}} \hat{v}^{3}\left(t, \frac{p}{3}\right)+G_{1}+G_{2},
\end{aligned}
$$

where $\lambda=\frac{x+y-2 z}{4 t}, \mu=\frac{y-x}{4 t}$,

$$
\begin{aligned}
G_{1}= & \frac{2 i \pi}{t \sqrt{3}(2 \pi)^{3 / 2}}\left(\frac{i p}{1+i p}\right)^{k+1} \iiint d x d y d z \\
& \times \exp \left(-2 i t p^{2} / 3-i p(x+y+z) / 3\right)\left(e^{-i\left(x^{2}+y^{2}+z^{2}-x y-x z-y z\right) / 6 t}-1\right) \\
& \times\left(1+\partial_{x}+\partial_{y}+\partial_{z}\right)^{k+1} v(t, x) v(t, y) v(t, z), \\
G_{2}= & \frac{2 \sqrt{3}}{(2 \pi)^{3 / 2}}\left(\frac{i p}{1+i p}\right)^{k+1} \iiint d x d y d z \exp \left(-2 i t p^{2} / 3-i p(x+y+z) / 3\right. \\
- & \left.i\left(x^{2}+y^{2}+z^{2}-x y-x z-y z\right) / 6 t\right) \\
& \quad \times \int_{p / 2 \sqrt{3}}^{\infty} d s \int_{-s \sqrt{3}}^{s \sqrt{3}} d q e^{2 i t(s-\lambda / \sqrt{3})^{2}+2 i t(q-\mu)^{2}} \\
& \quad \times\left(1+\partial_{x}+\partial_{y}+\partial_{z}\right)^{k+1} v(t, x) v(t, y) v(t, z) .
\end{aligned}
$$


Also we have

$$
\begin{aligned}
& Q_{2}=\frac{6 i p}{(2 \pi)^{3 / 2}} \iiint d x d y d z \exp \left(-2 i t p^{2}-i p(x+y-z)\right. \\
&\left.-i\left(z^{2}+x y-x z-y z\right) / 2 t\right) \int_{-\infty}^{-p / 3} d r \frac{e^{2 i t\left(r-\frac{2 p}{3}-\lambda\right)^{2}}}{1+\frac{p}{3}-2 r} \int_{p / 3+r}^{-p / 3-r} d q e^{-2 i t(q+\mu)^{2}} \\
& \quad \times\left(\partial_{x}+\partial_{y}+\partial_{z}\right)^{k} v(t, x) v(t, y)\left(v(t, z)-i v^{\prime}(t, z)\right) .
\end{aligned}
$$

And finally we obtain

$$
\begin{aligned}
& Q_{3}= \frac{6}{(2 \pi)^{3 / 2}} \iiint d x d y d z \exp (-i p(x+y-z)+i(x-z)(y-z) / 2 t) \\
& \times \iint d r d q e^{2 i t(q-\mu)^{2}-2 i t(r-2 p / 3+\lambda)^{2}}\left(\partial_{x}+\partial_{y}+\partial_{z}\right)^{k+1} v(t, x) v(t, y) v(t, z) \\
&- \frac{6 i p}{1+i p} \iiint d x d y d z e^{-i p(x+y-z)+i(x-z)(y-z) / 2 t} \int_{-\infty}^{p / 6} d r \\
& \times \int d q e^{2 i t(q-\mu)^{2}-2 i t(r-2 p / 3+\lambda)^{2}} \\
& \times\left(\partial_{x}+\partial_{y}+\partial_{z}\right)^{k}\left(1+\partial_{x}+\partial_{y}+\partial_{z}\right) v(t, x) v(t, y) v(t, z) \\
&-12 i p \iiint d x d y d z e^{-i p(x+y+z)+i(x-z)(y-z) / 2 t} \int_{p / 6}^{\infty} d r \\
& \quad \times \int_{p / 3+r}^{\infty} d q \frac{e^{2 i t(q-\mu)^{2}-2 i t(r-2 p / 3+\lambda)^{2}}}{1+p / 2+q} \\
& \quad \times\left(\partial_{x}+\partial_{y}+\partial_{z}\right)^{k}\left(1-i \partial_{y}-\frac{i}{2} \partial_{z}\right) v(t, x) v(t, y) v(t, z) \\
&(2.16) \quad \frac{3 \pi(i p)^{k+1}}{t}|\hat{v}(t, p)|^{2} \hat{v}(t, p)+G_{3}+G_{4}+G_{5},
\end{aligned}
$$

where $\lambda=\frac{x+y-2 z}{4 t}, \mu=\frac{y-x}{4 t}$,

$$
\begin{aligned}
G_{3}=\frac{3 \pi}{t} \iiint d x d y d z e^{-i p(x+y-z)}\left(e^{i(x-z)(y-z) / 2 t}-1\right) & \times\left(\partial_{x}+\partial_{y}+\partial_{z}\right)^{k+1} v(t, x) v(t, y) v(t, z), \\
G_{4}=- & \sqrt{\frac{\pi}{2 t}} \frac{6 i p e^{i \pi / 4}}{1+i p} \iiint d x d y d z \\
& \times \exp (-i p(x+y-z)+i(x-z)(y-z) / 2 t) \int_{-\infty}^{p / 6} d r e^{-2 i t(r-2 p / 3+\lambda)^{2}} \\
& \times\left(\partial_{x}+\partial_{y}+\partial_{z}\right)^{k}\left(1+\partial_{x}+\partial_{y}+\partial_{z}\right) v(t, x) v(t, y) v(t, z)
\end{aligned}
$$




$$
\begin{aligned}
& G_{5}=-12 i p \iiint d x d y d z \exp ^{(-i p(x+y-z)+i(x-z)(y-z) / 2 t)} \\
& \times \int_{p / 2}^{\infty} d q \int_{q-p / 3}^{\infty} d r \frac{e^{2 i t(q-\mu)^{2}-2 i t(r-2 p / 3+\lambda)^{2}}}{1+p / 2+q} \\
& \times\left(\partial_{x}+\partial_{y}+\partial_{z}\right)^{k}\left(1-i \partial_{x}-i \partial_{y}\right) v(t, x) v(t, y) v(t, z) .
\end{aligned}
$$

The remainder terms $G_{1}$ and $G_{3}$ can be easily estimated:

$$
\left\|G_{1}(t)\right\|_{\infty}+\left\|G_{3}(t)\right\|_{\infty} \leq C t^{-1-\gamma}\|v\|_{2,1}^{3},
$$

where $\gamma \in(0,1 / 4)$.

To estimate $G_{2}$ we prove the following estimate for all $t \geq 1, p>0 ; x, y, z \in \mathbf{R}$ :

$$
\begin{aligned}
A_{1} & =\left|\frac{p}{1+i p}\right|^{k+1}\left|\int_{p / 2 \sqrt{3}}^{\infty} d s \int_{-s \sqrt{3}}^{s \sqrt{3}} d q e^{2 i t(s-\lambda / \sqrt{3})^{2}+2 i t(q-\mu)^{2}}\right| \\
& \leq C t^{-1-\gamma}(1+|x|+|y|+|z|)^{2 \gamma} .
\end{aligned}
$$

We make the change of variables $s-\lambda / \sqrt{3}=s^{\prime} / \sqrt{2 t}, q-\mu=q^{\prime} / \sqrt{2 t}$. We get (the prime is omitted for simplicity)

$$
\begin{aligned}
A_{1} & =\frac{1}{2 t}\left|\frac{p}{1+i p}\right|^{k+1}\left|\int_{p \sqrt{t / 6}-\lambda \sqrt{2 t}}^{\infty} d s e^{i s^{2}} \int_{-s \sqrt{3}-(\lambda+\mu) \sqrt{2 t}}^{s \sqrt{3}+(\lambda-\mu) \sqrt{2 t}} d q e^{i q^{2}}\right| \\
& =\frac{\pi}{2 t}\left|\frac{p}{1+i p}\right|^{k+1}\left|\int_{\eta}^{\infty} d s e^{i s^{2}}(\bar{E}(a s+b)-\bar{E}(-a s+\chi))\right|,
\end{aligned}
$$

where $\eta=p \sqrt{t / 6}-\lambda \sqrt{2 t}, a=\sqrt{3}, b=(\lambda-\mu) \sqrt{2 t}, \chi=-(\lambda+\mu) \sqrt{2 t}$. Now we use the estimate

$$
\begin{aligned}
& p \sqrt{t / 6}=\eta+\lambda \sqrt{2 t} \leq \max (1, \eta)+\lambda \sqrt{2 t} \leq \max (1, \eta)(1+|\lambda \sqrt{2 t}|) \\
& \quad \leq \max (1, \eta)(1+|x|+|y|+|z|) .
\end{aligned}
$$

Therefore by Lemma 2.3 we obtain

$$
\begin{aligned}
A_{1} \leq & C t^{-1-\gamma}(1+|x|+|y|+|z|)^{2 \gamma}(\max (1, \eta)) \\
& \times\left(\left|\int_{\eta}^{\infty} e^{i s^{2}} \bar{E}(a s+b) d s\right|+\left|\int_{\eta}^{\infty} e^{i s^{2}} \bar{E}(-a s+\chi) d s\right|\right) \\
\leq & C t^{-1-\gamma}(1+|x|+|y|+|z|)^{2 \gamma},
\end{aligned}
$$

so we get (2.18). And from (2.18) we obtain an estimate of the second remainder $G_{2}$ in the representation (2.15):

$$
\left\|G_{2}(t)\right\|_{\infty} \leq C t^{-1-\gamma}\|v\|_{2,1}^{3}
$$

To estimate the integral $Q_{2}$ we prove the following estimate:

$$
\begin{aligned}
A_{2} & =p\left|\int_{-\infty}^{-p / 3} d r \frac{e^{2 i t(r-2 p / 3-\lambda)^{2}}}{1+\frac{p}{3}-2 r} \int_{p / 3+r}^{-p / 3-r} d q e^{-2 i t(q+\mu)^{2}}\right| \\
& \leq C t^{-1-\gamma}(1+|x|+|y|+|z|)^{2 \gamma}
\end{aligned}
$$

We now make the change of variables $(r-2 p / 3-\lambda) \sqrt{2 t}=-r^{\prime},(q+\mu) \sqrt{2 t}=q^{\prime}$. Then we obtain, again omitting the prime,

$$
A_{2}=\frac{\pi p}{2 t}\left|\int_{\eta}^{\infty} d r \frac{e^{i r^{2}}}{1+2 p / 3+(r-\eta) / \sqrt{2 t}}\left(-\bar{E}\left(r+b_{1}\right)+\bar{E}\left(-r+b_{2}\right)\right)\right|,
$$


where

$$
\begin{aligned}
\eta & =(p+\lambda) \sqrt{2 t}=\left(p+\frac{x+y-2 z}{4 t}\right) \sqrt{2 t}, b_{1}=-(p+\lambda-\mu) \sqrt{2 t} \\
& =-\left(p+\frac{x-z}{2 t}\right) \sqrt{2 t}, b_{2}=(p+\lambda+\mu) \sqrt{2 t}=\left(p+\frac{y-z}{2 t}\right) \sqrt{2 t} .
\end{aligned}
$$

Since

$$
p \sqrt{t} \leq C \max \left(1, \min \left(\eta,\left|b_{1}\right|,\left|b_{2}\right|\right)\right)(1+|x|+|y|+|z|),
$$

and so

$$
p \leq C(1+p)^{1-2 \gamma} t^{-\gamma}(1+|x|+|y|+|z|)^{2 \gamma} \max \left(1, \min \left(\eta,\left|b_{1}\right|,\left|b_{2}\right|\right)\right)^{2 \gamma}
$$

we can apply Lemma 2.4 to obtain estimate (2.20). From (2.20) we can easily get

$$
\left\|Q_{2}\right\|_{\infty} \leq C t^{-1-\gamma}\|v\|_{2,1}^{3} \text {. }
$$

Consider the integral $G_{4}$. We prove the following estimate:

$$
A_{3}=\frac{p}{|i p+1|}\left|\int_{-\infty}^{p / 6} d r e^{-2 i t(r-2 p / 3+\lambda)^{2}}\right| \leq C t^{-1 / 2-\gamma}(1+|x|+|y|+|z|)^{2 \gamma}
$$

We make the change of variables $p / 6-r=r^{\prime}, \lambda-p / 2=\frac{x+y-2 z}{4 t}-p / 2=\lambda^{\prime}$. Then we get, omitting the prime,

$$
A_{3}=\frac{p}{|i p+1|}\left|\int_{0}^{\infty} d r e^{-2 i t(r-\lambda)^{2}}\right| .
$$

We consider the two possible cases: 1) $\left|\frac{x+y-2 z}{4 t}\right|<p / 4$; then $\lambda<-p / 4$, and integration by parts yields

$$
A_{3}=\frac{p}{|i p+1|}\left|\frac{e^{-2 i t \lambda^{2}}}{4 i t \lambda}+\int_{0}^{\infty} \frac{e^{-2 i t(r-\lambda)^{2}} d r}{4 i t(r-\lambda)^{2}}\right| \leq C / t
$$

2) $\left|\frac{x+y-2 z}{4 t}\right| \geq p / 4$; then we have

$$
A_{3}=\frac{p \pi}{\sqrt{2 t}|i p+1|}|E(-\lambda \sqrt{2 t})| \leq C p^{\gamma} t^{-1 / 2} \leq C t^{-1 / 2-\gamma}|x+y-2 z|^{\gamma} .
$$

Thus the estimate (2.23) is true. Using (2.23), we easily get

$$
\left\|G_{4}\right\|_{\infty} \leq C t^{-1-\gamma}\|v\|_{2,1}^{3} \text {. }
$$

To estimate the last remainder $G_{5}$, let us prove the following estimate:

$$
A_{4}=p\left|\int_{p / 2}^{\infty} d q \int_{q-p / 3}^{\infty} d r \frac{e^{2 i t(q-\mu)^{2}-2 i t(r-2 p / 3+\lambda)^{2}}}{1+p / 2+q}\right| \leq C t^{-1-\gamma}(1+|x|+|y|+|z|)^{\gamma} .
$$

We make the change of variables $(q-\mu) \sqrt{2 t}=q^{\prime},(r-2 p / 3+\lambda) \sqrt{2 t}=r^{\prime}$; then we have, omitting the prime,

$$
A_{4}=\frac{\pi p}{2 t}\left|\int_{\eta}^{\infty} \frac{e^{i q^{2}} \bar{E}(q+\chi) d q}{1+p+(q-\eta) / \sqrt{2 t}}\right|
$$

where $\eta=(p / 2-\mu) \sqrt{2 t}, \chi=(\mu+\lambda-p) \sqrt{2 t}$. Then using (2.21) and Lemma 2.4 we get (2.25). And from (2.25) we obtain easily

$$
\left\|G_{5}\right\|_{\infty} \leq C t^{-1-\gamma}\|v\|_{2,1}^{3} .
$$


Substituting representations (2.15), (2.16) and estimates (2.17), (2.19), (2.22), $(2.24),(2.26)$ into $(2.11)$, we get the result of the lemma. Lemma 2.5 is proved.

\section{Proofs of Theorems 1.1 And 1.2}

We define the function space $X_{T}$ as follows:

$$
\begin{aligned}
X_{T}=\left\{\phi \in C\left([0, T] ; \mathcal{S}^{\prime}\right) ;\right. & \|\phi\|\left\|_{X_{T}}=\sup _{t \in[0, T]}\right\| \phi(t) \|_{2,0} \\
& \left.+\sup _{t \in[0, T]}\|U(-t) \phi(t)\|_{1,1}<\infty\right\} .
\end{aligned}
$$

To clarify the idea of the proof of the theorems we only show a priori estimates of local solutions to the BO equation. For that purpose we assume that the following local existence theorem holds.

Theorem 3.1. Assume that $\left\|u_{0}\right\|_{2,0}+\left\|u_{0}\right\|_{1,1}=\epsilon \leq \epsilon^{\prime}$ and $\epsilon^{\prime}$ is sufficiently small. Then there exist a finite time interval $[0, T]$ with $T>1$ and a unique solution $u$ of (1.1) with $\rho>3$ such that

$$
\|u\| \|_{X_{T}} \leq C \epsilon^{\prime}
$$

For the proof of Theorem 3.1, see, e.g, [1, 2, 13, 18, 19, 24, 28, 30, 31].

Lemma 3.1. Let $u$ be the local solution of the Cauchy problem (1.1) with $\rho>3$ that exists by Theorem 3.1. Then, for any $t \in[0, T]$,

$$
\|u(t)\|_{2,0}+\|U(-t) u(t)\|_{1,1} \leq C \epsilon
$$

Proof. Denote

$$
L u=\left(\partial_{t}+\mathcal{H} \partial_{x}^{2}\right) u
$$

Then we can write down the $\mathrm{BO}$ equation (1.1) as

$$
L u=-\left(|u|^{\rho-1} u\right)_{x} .
$$

First of all we note that the conservation law

$$
\|u\|=\left\|u_{0}\right\|
$$

holds. Then we differentiate equation (1.1) twice with respect to $x$ to get

$$
L u_{x x}=-\left(|u|^{\rho-1} u\right)_{x x x} .
$$

Multiplying both sides of this equation by $u_{x x}$ and integrating by parts, we obtain

$$
\frac{d}{d t}\left\|u_{x x}\right\|^{2} \leq C\left(\|u\|_{\infty}+\left\|u_{x}\right\|_{\infty}\right)^{\rho-1}\left(\left\|u_{x x}\right\|^{2}+\|u\|^{2}\right) .
$$

By Sobolev's inequality (Lemma 2.1) and Theorem 3.1 we have

$$
\|u\|_{\infty}+\left\|u_{x}\right\|_{\infty} \leq C\|u\|_{2,0} \leq C \epsilon^{\prime}
$$

for $t \leq 1$, and using estimate (2.1) of Lemma 2.2 and Theorem 3.1 we get

$$
\|u\|_{\infty}+\left\|u_{x}\right\|_{\infty} \leq C(1+|t|)^{-1 / 2}\|U(-t) u\|_{2,1} \leq C \epsilon^{\prime} t^{-1 / 2} .
$$

Therefore from (3.1), (3.2) and the Gronwall inequality we obtain

$$
\left\|u_{x x}\right\|^{2} \leq C\left\|u_{0 x x}\right\|^{2} \leq C \epsilon^{2} .
$$

In the same way as in the proof of the above inequality we get

$$
\|u\|_{2,0} \leq C \epsilon .
$$


Let us define the dilation operator $I \phi=x \phi+2 t \int_{-\infty}^{x} \partial_{t} \phi d x^{\prime}$ and the operator $J \phi=x \phi-2 t \int_{-\infty}^{x} \mathcal{H} \partial_{x}^{2} \phi d x^{\prime}=x \phi-2 t \mathcal{H} \partial_{x} \phi$. Their difference is equal to

$$
I \phi-J \phi=2 t \int_{-\infty}^{x} L \phi d x^{\prime} .
$$

Also we note that the commutator representations

$$
[L, J] \phi=0, \quad[L, I] \phi=2 \int_{-\infty}^{x} L \phi d x^{\prime}, \quad\left[J, \partial_{x}\right] \phi=\left[I, \partial_{x}\right] \phi=-\phi
$$

are valid. Using (3.5) we get

$$
\begin{aligned}
I\left(|u|^{\rho-1} u\right)_{x} & =x \partial_{x}\left(|u|^{\rho-1} u\right)+2 t \partial_{t}\left(|u|^{\rho-1} u\right) \\
& =\rho|u|^{\rho-1}\left(x \partial_{x} u+2 t \partial_{t} u\right)=\rho|u|^{\rho-1} I u_{x} .
\end{aligned}
$$

Therefore, applying the operator I to the equation (1.1), we find that

$$
\begin{aligned}
L I u & =I L u+2 \int_{-\infty}^{x} L u d x^{\prime}=-I\left(|u|^{\rho-1} u\right)_{x}-2|u|^{\rho-1} u \\
& =-\rho|u|^{\rho-1} I u_{x}-2|u|^{\rho-1} u=-\rho|u|^{\rho-1}(I u)_{x}-(2-\rho)|u|^{\rho-1} u .
\end{aligned}
$$

Multiplying (3.7) by $I u$ and integrating by parts, we get

$$
\frac{d}{d t}\|I u\|^{2} \leq C\left(\|u\|_{\infty}+\left\|u_{x}\right\|_{\infty}\right)^{\rho-1}\left(\|I u\|^{2}+\|I u\|\|u\|\right) .
$$

Analogously we have

$$
\begin{aligned}
L I u_{x} & =I L u_{x}+2 L u=-I\left(|u|^{\rho-1} u\right)_{x x}-2\left(|u|^{\rho-1} u\right)_{x} \\
& =-\left(I\left(|u|^{\rho-1} u\right)_{x}\right)_{x}-\left(|u|^{\rho-1} u\right)_{x} \\
& =-\rho\left(|u|^{\rho-1}\left(I u_{x}\right)_{x}-(\rho-1)|u|^{\rho-3} u u_{x} I u_{x}-|u|^{\rho-1} u_{x}\right) .
\end{aligned}
$$

Multiplying (3.9) by $I u_{x}$ and integrating by parts, we obtain

$$
\frac{d}{d t}\left\|I u_{x}\right\|^{2} \leq C\left(\|u\|_{\infty}+\left\|u_{x}\right\|_{\infty}\right)^{\rho-1}\left(\left\|I u_{x}\right\|^{2}+\|u\|\left\|I u_{x}\right\|\right) .
$$

Applying (3.2), (3.3) and the Gronwall inequality to (3.8) and (3.10), we obtain the estimate

$$
\|I u\|+\left\|I u_{x}\right\| \leq C\left(\left\|x u_{0}\right\|+\left\|x u_{0 x}\right\|\right) \leq C \epsilon,
$$

whence by (3.2), (3.3) and the identity (3.4) we get

$$
\|J u\|+\left\|J u_{x}\right\| \leq\|I u\|+\left\|I u_{x}\right\|+C t\left(\left\||u|^{\rho-1} u\right\|+\left\||u|^{\rho-1} u_{x}\right\|\right) \leq C \epsilon .
$$

The lemma follows immediately by applying (3.3) and (3.11), since we have

$$
\|J U(t) f\|=\|\mathcal{F} J U(t) f\|=\|x f\|,
$$

from which it follows that

$$
\|J u\|=\|J U(t) U(-t) u\|=\|x U(-t) u\| .
$$

We are now in a position to prove Theorems 1.1 and 1.2 . 
Proof of Theorem 1.1. We have by Lemma 3.1

$$
\|\| u \|_{X_{T}} \leq C \epsilon \text { for } t \in[0, T] .
$$

We take $\epsilon$ satisfying $C \epsilon \leq \epsilon^{\prime}$. Then a standard continuation argument yields the result because our constants $C$ do not depend on the time $T$ of existence of solutions.

Proof of Theorem 1.2. We have by (1.1)

$$
\left(U(-t) \partial_{x}^{j} u\right)_{t}=-U(-t) \partial_{x}^{j+1}\left(|u|^{\rho-1} u\right), \quad j=0,1,
$$

and by $(3.7)$

$$
(U(-t) I u)_{t}=-U(-t)\left(\rho|u|^{\rho-1}(I u)_{x}+(2-\rho)|u|^{\rho-1} u\right) .
$$

Hence by (3.2) we get

$$
\begin{gathered}
\left.\left\|U(-t)\left(\partial_{x}^{j} u\right)(t)-U(-s)\left(\partial_{x}^{j} u\right)(s)\right\| \leq C \int_{s}^{t}\left(\|u(\tau)\|_{\infty}+\left\|u_{x}(\tau)\right\|\right)^{\rho-1}\right)\|u(\tau)\|_{2,0} d \tau \\
\leq C \epsilon \int_{s}^{t} \tau^{-(\rho-1) / 2} d \tau \leq C \epsilon\left(s^{-(\rho-3) / 2}+t^{-(\rho-3) / 2}\right)
\end{gathered}
$$

and by (3.2) and (3.11)

$$
\begin{gathered}
\|U(-t) I u(t)-U(-s) I u(s)\| \leq C \int_{s}^{t}\|u(\tau)\|_{\infty}^{\rho-1}\left(\left\|I u_{x}(\tau)\right\|+\|u(\tau)\|\right) d \tau \\
\leq C \epsilon \int_{s}^{t} \tau^{-(\rho-1) / 2} d \tau \leq C \epsilon\left(s^{-(\rho-3) / 2}+t^{-(\rho-3) / 2}\right) .
\end{gathered}
$$

From (3.4) and (3.2) it follows that

$$
\begin{gathered}
\|U(-t)(I-J) u(t)\| \leq C t\left\|\int_{-\infty}^{x} \partial_{x^{\prime}}\left(|u|^{\rho-1} u\right) d x^{\prime}\right\| \\
\leq C t\|u(t)\|_{\infty}^{\rho-1}\|u(t)\| \leq C \epsilon t^{-\frac{\rho-3}{2}} .
\end{gathered}
$$

By (3.12)-(3.14) we find that there exists a unique function $V \in H^{1,0} \cap H^{0,1}$ such that

$$
\lim _{t \rightarrow \infty}\left(\left\|U(-t) u(t)-\mathcal{F}^{-1} V\right\|_{1,0}+\left\|U(-t) u(t)-\mathcal{F}^{-1} V\right\|_{0,1}\right)=0 .
$$

This implies (1.2). By Hölder's inequality and (1.2)

$$
\begin{aligned}
\|\mathcal{F}(U(-t) u)(t)-V\|_{\infty} & =\left\|\mathcal{F}(U(-t) u)(t)-\mathcal{F F}^{-1} V\right\|_{\infty} \\
& \leq C\left\|U(-t) u(t)-\mathcal{F}^{-1} V\right\|_{1} \\
& \leq C\left\|U(-t) u(t)-\mathcal{F}^{-1} V\right\|_{0,1} \rightarrow 0 \quad \text { as } \quad t \rightarrow \infty
\end{aligned}
$$

The asymptotic formula (1.3) follows from (2.4), (3.15), and (2.5). This completes the proof of Theorem 1.2. 


\section{Proofs of Theorems 1.3 And 1.4}

We define the function space $Y_{T}$ as follows:

$$
\begin{aligned}
Y_{T}=\left\{\phi \in C\left([0, T] ; \mathcal{S}^{\prime}\right):\|\phi\| \|_{Y_{T}}\right. & =\sup _{t \in[0, T]}(1+|t|)^{-C \epsilon}\|\phi(t)\|_{3,0} \\
& +\sup _{t \in[0, T]}(1+|t|)^{-C \epsilon}\|U(-t) \phi(t)\|_{2,1} \\
& \left.+\sup _{t \in[0, T]} \sqrt{1+|t|}\left(\|\phi(t)\|_{\infty}+\left\|\phi_{x}(t)\right\|_{\infty}\right)<\infty\right\},
\end{aligned}
$$

where $\epsilon$ depends only on the size of the initial function.

We first state the local existence theorem without a proof.

Theorem 4.1. Assume that $\left\|u_{0}\right\|_{3,0}+\left\|u_{0}\right\|_{1,2}=\epsilon \leq \epsilon^{\prime}$ and $\epsilon^{\prime}$ is sufficiently small. Then there exist a finite time interval $[0, T]$ with $T>1$ and a unique solution $u$ of (1.1) with $\rho=3$ such that

$$
\|u\| \|_{Y_{T}} \leq C \epsilon^{\prime}
$$

For the proof of Theorem 4.1, see, e.g, [14, 15, 20, 29, 35].

Lemma 4.1. Let $u$ be the local solution of the Cauchy problem (1.1) with $\rho=3$ that exists by Theorem 4.1. Then, for any $t \in[0, T]$,

$$
(1+|t|)^{-C \epsilon^{\prime}}\left(\|u(t)\|_{3,0}+\|U(-t) u(t)\|_{2,1}\right) \leq C \epsilon .
$$

Proof. Analogously to the proof of Lemma 3.1, we differentiate equation (1.1) three times with respect to $x$, multiply the result by $u_{x x x}$ and integrate by parts. Then we have

$$
\begin{aligned}
\frac{d}{d t}\left\|u_{x x x}\right\|^{2} & \leq C\left(\|u\|_{\infty}\left\|u_{x}\right\|_{\infty}\left\|u_{x x x}\right\|^{2}+\left\|u_{x}\right\|_{\infty}^{2}\left\|u_{x x}\right\|\left\|u_{x x x}\right\|\right) \\
& \leq C\left(\epsilon^{\prime}\right)^{2}(1+|t|)^{-1}\left(\left\|u_{x x x}\right\|^{2}+\left\|u_{x x}\right\|^{2}\right)
\end{aligned}
$$

since

$$
\|u\|_{\infty}+\left\|u_{x}\right\|_{\infty} \leq C \epsilon^{\prime}(1+|t|)^{-1 / 2}
$$

Similarly,

$$
\frac{d}{d t}\|u\|_{3,0}^{2} \leq C\left(\epsilon^{\prime}\right)^{2}(1+|t|)^{-1}\|u\|_{3,0}^{2} .
$$

Therefore using the Gronwall inequality we get

$$
\|u\|_{3,0} \leq C \epsilon(1+|t|)^{C \epsilon^{\prime}} .
$$

Now using (3.4), (3.5) and (4.1) we find that

$$
\begin{aligned}
\|J u\| & \leq\|I u\|+\left\|2 t \int_{-\infty}^{x} L u d x^{\prime}\right\| \\
& \leq\|I u\|+C|t|\left\|u^{3}\right\| \leq\|I u\|+C\left(\epsilon^{\prime}\right)^{2}\|u\|, \\
\left\|(J u)_{x}\right\| & \leq\left\|I u_{x}\right\|+\|u\|+\|2 t L u\| \leq\left\|I u_{x}\right\|+\|u\|+C\left(\epsilon^{\prime}\right)^{2}\|u\|, \\
\left\|(J u)_{x x}\right\|^{2} & \leq\left|\left(J u_{x x x}, I u_{x}\right)\right|+C\left\|I u_{x}\right\|\left\|u_{x x}\right\|+C\left\|u_{x}\right\|^{2} \\
& \leq C\left(\|u\|_{3,0}^{2}+\left\|I u_{x}\right\|^{2}+\left\|J I u_{x}\right\|^{2}\right)\left(1+(1+|t|)\|u\|_{\infty}^{2}\right) \\
& \leq C\left(\left(\epsilon^{\prime}\right)^{2}+1\right)\left(\|u\|_{3,0}^{2}+\left\|I u_{x}\right\|^{2}+\left\|J I u_{x}\right\|^{2}\right) .
\end{aligned}
$$


And since

$$
\int_{-\infty}^{x} L I u_{x} d x^{\prime}=\int_{-\infty}^{x} L(I u)_{x} d x^{\prime}-\int_{-\infty}^{x} L u d x^{\prime}=u^{3}+L I u=-u^{3}-3 u^{2} I u_{x},
$$

we obtain

$$
\begin{aligned}
\left\|J I u_{x}\right\| & \leq\left\|I^{2} u_{x}\right\|+\left\|2 t \int_{-\infty}^{x} L I u_{x} d x^{\prime}\right\| \\
& \leq\left\|I^{2} u_{x}\right\|+C|t|\left\|u^{3}\right\|+C|t|\left\|u^{2} I u_{x}\right\| \\
& \leq\left\|I^{2} u_{x}\right\|+C|t|\|u\|_{\infty}^{2}\left(\|u\|+\left\|I u_{x}\right\|\right) .
\end{aligned}
$$

Substituting (4.6) into (4.5), in view of (4.3) and (4.4) we get

$$
\|x U(-t) u\|_{2,0}^{2}=\|J u\|_{2,0}^{2} \leq C\left(\left(\epsilon^{\prime}\right)^{2}+1\right)\left(\|u\|_{3,0}^{2}+\|I u\|^{2}+\left\|I u_{x}\right\|^{2}+\left\|I^{2} u_{x}\right\|^{2}\right) .
$$

Now we use estimates (3.8), (3.10); then via (4.1) and the Gronwall inequality we easily have

$$
\|I u\|+\left\|I u_{x}\right\| \leq C \epsilon(1+|t|)^{C \epsilon^{\prime}} .
$$

Let us estimate $\left\|I^{2} u_{x}\right\|$. Using (3.5), we find that

$$
\begin{aligned}
L I^{2} u_{x} & =I L I u_{x}+2 \int_{-\infty}^{x} L I u_{x} d x^{\prime} \\
& =I^{2} L u_{x}-2 I\left(u^{3}\right)_{x}-2 \int_{-\infty}^{x} I\left(u^{3}\right)_{x x} d x^{\prime}-4 u^{3} \\
& =I^{2} L u_{x}-4 I\left(u^{3}\right)_{x}-2 u^{3}=-I^{2}\left(u^{3}\right)_{x x}-4 I\left(u^{3}\right)_{x}-2 u^{3} .
\end{aligned}
$$

Then an easy computation gives us

$$
I(\phi \psi)_{x}=\phi I \psi_{x}+\psi I \phi_{x}
$$

therefore $I\left(u^{3}\right)_{x}=3 u^{2} I u_{x}$ and

$$
\begin{aligned}
I^{2}\left(u^{3}\right)_{x x} & =I\left(I\left(u^{3}\right)_{x}\right)_{x}-I\left(u^{3}\right)_{x}=3 I\left(\left(u^{2} I u_{x}\right)_{x}\right)-3 u^{2} I u_{x} \\
& =6 u\left(I u_{x}\right)^{2}+3 u^{2} I\left(I u_{x}\right)_{x}-3 u^{2} I u_{x}=6 u\left(I u_{x}\right)^{2}+3 u^{2}\left(I^{2} u_{x}\right)_{x}-6 u^{2} I u_{x} .
\end{aligned}
$$

Thus $L I^{2} u_{x}=-6 u\left(I u_{x}\right)^{2}-3 u^{2}\left(I^{2} u_{x}\right)_{x}-6 u^{2} I u_{x}-2 u^{3}$. Multiplying this equation by $I^{2} u_{x}$ and integrating by parts, we get

$$
\begin{aligned}
\frac{d}{d t}\left\|I^{2} u_{x}\right\|^{2} & \leq-2\left(I^{2} u_{x},\left(6 u\left(I u_{x}\right)^{2}+3 u^{2}\left(I^{2} u_{x}\right)_{x}+6 u^{2} I u_{x}+2 u^{3}\right)\right) \\
& \leq C\left(\|u\|_{\infty}+\left\|u_{x}\right\|_{\infty}\right)^{2}\left(\left\|I^{2} u_{x}\right\|^{2}+\left\|I u_{x}\right\|^{2}+\|u\|^{2}\right) \\
& +C\|u\|_{\infty}\left\|I u_{x}\right\|_{\infty}\left\|I^{2} u_{x}\right\|\left\|I u_{x}\right\| .
\end{aligned}
$$

From the identity (2.3) and the Hölder inequality we have, for $0<\gamma<1 / 2$,

$$
\|\phi\|_{\infty} \leq C t^{-1 / 2}\|U(-t) \phi\|_{1} \leq C t^{-1 / 2}\left(\|\phi\|+\|\phi\|^{1 / 2-\gamma}\|J \phi\|^{1 / 2+\gamma}\right),
$$

whence via (4.2), (4.8) and (4.6) we get

$$
\begin{aligned}
\left\|I u_{x}\right\|_{\infty} & \leq C t^{-1 / 2}\left(\left\|I u_{x}\right\|+\left\|I u_{x}\right\|^{1 / 2-\gamma}\left\|J I u_{x}\right\|^{1 / 2+\gamma}\right) \\
& \leq C t^{-1 / 2}\left(\left\|I u_{x}\right\|+\left\|I u_{x}\right\|^{1 / 2-\gamma}\left\|I^{2} u_{x}\right\|^{1 / 2+\gamma}\right) \\
& \leq C t^{-1 / 2}\left(\left\|I u_{x}\right\|+\left\|I u_{x}\right\|^{2 /(1-2 \gamma)}+\left\|I^{2} u_{x}\right\|\right)
\end{aligned}
$$


By virtue of (4.1), (4.8) and (4.10) we find from (4.9) that

$$
\begin{aligned}
\frac{d}{d t}\left\|I^{2} u_{x}\right\|^{2} & \leq C\left(\epsilon^{\prime}\right)^{2} t^{-1}\left\|I^{2} u_{x}\right\|^{2}+C \epsilon^{2} t^{-1+C \epsilon^{\prime}} \\
& +C \epsilon^{\prime} t^{-1}\left(\left\|I u_{x}\right\|^{2}+\left\|I u_{x}\right\|^{(3-2 \gamma) /(1-2 \gamma)}+\left\|I^{2} u_{x}\right\|\right)\left\|I^{2} u_{x}\right\| \\
& \leq C \epsilon^{\prime} t^{-1}\left\|I^{2} u_{x}\right\|^{2}+C \epsilon^{2} t^{-1+C \epsilon^{\prime}}
\end{aligned}
$$

Applying the Gronwall inequality, we get

$$
\left\|I^{2} u_{x}\right\|^{2} \leq C \epsilon^{2}(1+|t|)^{C \epsilon^{\prime}} .
$$

Estimates (4.2), (4.7), (4.8) and (4.11) give us the result. Lemma 4.1 is proved.

Lemma 4.2. Let $u$ be the local solution of the Cauchy problem (1.1) with $\rho=3$ that exists by Theorem 4.1. Then for any $t \in[0, T]$ we have

$$
\sqrt{1+t}\left(\|u(t)\|_{\infty}+\left\|u_{x}(t)\right\|_{\infty}\right) \leq C \epsilon .
$$

Proof. By Lemma 4.1 we have

$$
\sqrt{1+|t|}\left(\|u(t)\|_{\infty}+\left\|u_{x}(t)\right\|_{\infty}\right) \leq C \epsilon \quad \text { for } \quad t \leq 1 .
$$

We assume that $t \geq 1$. From Lemma 2.2 and Lemma 4.1 it follows that

$$
\begin{aligned}
& \|u(t)\|_{\infty}+\left\|u_{x}(t)\right\|_{\infty} \\
& \quad \leq C \epsilon t^{-1 / 2-\alpha+C \epsilon^{\prime}}+C t^{-1 / 2}\left(\|\mathcal{F} U(-t) u(t)\|_{\infty}+\left\|\mathcal{F} U(-t) u_{x}(t)\right\|_{\infty}\right) .
\end{aligned}
$$

We estimate the second summand in the right-hand side of inequality (4.13). Multiplying both sides of (1.1) by $U(-t)$, we obtain

$$
(U(-t) u(t))_{t}+U(-t)\left(u^{3}\right)_{x}=0 .
$$

We define $v(t)=U(-t) u(t)$; then, taking the Fourier transform, we get

$$
\hat{v}_{t}(t, p)+i p \iint d \xi_{1} d \xi_{2} e^{i t \mathcal{L}} \hat{v}\left(t, \xi_{1}\right) \hat{v}\left(t, \xi_{2}\right) \hat{v}\left(t, \xi_{3}\right)=0,
$$

where $\xi_{3}=p-\xi_{1}-\xi_{2}, \mathcal{L}=-p|p|+\xi_{1}\left|\xi_{1}\right|+\xi_{2}\left|\xi_{2}\right|+\xi_{3}\left|\xi_{3}\right|$. Since the solution $v(t, x)$ is real, $\hat{v}(t,-p)=\overline{\hat{v}(t, p)}$. Therefore it is sufficient to consider only $p>0$. By Lemma 2.5 we have the following equation for the function $\hat{v}(t, p)$ for $p>0, \quad t>0, \quad k=$ 0,1 :

$$
\begin{aligned}
(i p)^{k} \hat{v}_{t}(t, p) & +(i p)^{k+1} \frac{i \pi e^{-2 i t p^{2} / 3}}{t \sqrt{3}} \hat{v}^{3}\left(t, \frac{p}{3}\right) \\
& +\frac{3(i p)^{k+1} \pi}{t}|\hat{v}(t, p)|^{2} \hat{v}(t, p)+O\left(t^{-1-\gamma}\|v\|_{2,1}^{3}\right)=0 .
\end{aligned}
$$

To get rid of the third summand in the left-hand side of equation (4.16) we make a change of the dependent variable: $\hat{v}=\hat{w} B$, where

$$
B(t)=\exp \left(-3 i p \pi \int_{1}^{t}|\hat{v}(\tau, p)|^{2} \frac{d \tau}{\tau}\right) .
$$


Then integrating (4.16) with respect to $t$ from 1 to $t$ and using Lemma 4.1, we obtain

$$
\begin{aligned}
(i p)^{k} \hat{w}(t) & =(i p)^{k} \hat{w}(1)-\frac{i(i p)^{k+1} \pi}{\sqrt{3}} \int_{1}^{t} B(\tau) e^{-2 i \tau p^{2} / 3} \hat{v}^{3}\left(\tau, \frac{p}{3}\right) \frac{d \tau}{\tau} \\
& +C \epsilon \int_{1}^{t} \tau^{-1-\gamma+C \epsilon^{\prime}} d \tau .
\end{aligned}
$$

Hence we get

$$
\begin{aligned}
& \|\mathcal{F} U(-t) u(t)\|_{\infty}+\left\|\mathcal{F} U(-t) u_{x}(t)\right\|_{\infty}=\|(1+|p|) \hat{v}\|_{\infty}=\|(1+|p|) \hat{w}\|_{\infty} \\
& \quad \leq C \epsilon+C p(1+|p|)\left|\int_{1}^{t} B(\tau) e^{-2 i \tau p^{2} / 3} \hat{v}^{3}\left(\tau, \frac{p}{3}\right) \frac{d \tau}{\tau}\right| .
\end{aligned}
$$

To estimate the last integral in (4.18) we integrate by parts, using the identity

$$
e^{-2 i \tau p^{2} / 3}=\frac{1}{1-2 i \tau p^{2} / 3} \partial_{\tau}\left(\tau e^{-2 i \tau p^{2} / 3}\right)
$$

Then for $1 \leq s \leq t$ and $p>0$ we have

$$
\begin{aligned}
p(1 & +p)\left|\int_{s}^{t} B(\tau) e^{-2 i \tau p^{2} / 3} \hat{v}^{3}\left(\tau, \frac{p}{3}\right) \frac{d \tau}{\tau}\right| \\
& =p(1+p) \mid\left[\frac{B(\tau) e^{-2 i \tau p^{2} / 3} \hat{v}^{3}\left(\tau, \frac{p}{3}\right)}{1-2 i \tau p^{2} / 3}\right]_{s}^{t}-\int_{s}^{t} \frac{B(\tau) e^{-2 i \tau p^{2} / 3}}{1-2 i \tau p^{2} / 3}\left(\frac{\hat{v}^{3}\left(\tau, \frac{p}{3}\right) 2 i p^{2} / 3}{1-2 i \tau p^{2} / 3}\right. \\
& \left.+3 \hat{v}^{2}\left(\tau, \frac{p}{3}\right) \hat{v}_{\tau}\left(\tau, \frac{p}{3}\right)-\frac{3 i p \pi}{\tau} \hat{v}^{3}\left(\tau, \frac{p}{3}\right)|\hat{v}(\tau, p)|^{2}\right) \frac{d \tau}{\tau} \mid .
\end{aligned}
$$

Using the Sobolev inequality (Lemma 2.1) and Lemma 4.1, we obtain the rough estimate

$$
\|(1+|p|) \hat{v}\|_{\infty} \leq\|v\|_{1}+\left\|v_{x}\right\|_{1} \leq\|v\|_{2,1} \leq C \epsilon(1+|t|)^{C \epsilon^{\prime}}
$$

and from (4.16) we have, for $\hat{v}_{t}(t, p)$,

$$
\left\|(i p)^{k} \hat{v}_{t}(t, p)\right\|_{\infty} \leq C \epsilon t^{C \epsilon^{\prime}-1} .
$$

Substituting (4.20) and (4.21) into (4.19), we get

$$
p(1+p)\left|\int_{s}^{t} B(\tau) e^{-2 i \tau p^{2} / 3} \hat{v}^{3}\left(\tau, \frac{p}{3}\right) \frac{d \tau}{\tau}\right| \leq C \epsilon s^{-\gamma+C \epsilon^{\prime}} .
$$

Estimates (4.13), (4.18) and (4.22) gives us the desired result. Lemma 4.2 is proved.

In the same way as in the proof of Lemma 4.1, we have by Lemma 4.2

Lemma 4.3. Let $u$ be the local solution of the Cauchy problem (1.1) with $\rho=3$ that exists by Theorem 4.1. Then for any $t \in[0, T]$ we have

$$
(1+|t|)^{-C \epsilon}\left(\|u(t)\|_{3,0}+\|U(-t) u(t)\|_{2,1}\right) \leq C \epsilon .
$$

We are now in a position to prove Theorems 1.3 and 1.4.

Proof of Theorem 1.3. In the same way as in the proof of Theorem 1.1 we have Theorem 1.3, by Lemmas 4.1 and 4.3. 
Proof of Theorem 1.4. From equation (4.17) and estimate (4.22) we have

$$
\|\hat{w}(t)-\hat{w}(s)\|_{\infty} \leq C \epsilon s^{-\gamma+C \epsilon} .
$$

Hence there exists a unique limit function $W \in L^{\infty}$ such that

$$
\|W-\hat{w}(t)\|_{\infty} \leq C \epsilon t^{-\gamma+C \epsilon}
$$

This implies estimate (1.5) in Theorem 1.4. We now let

$$
\left.\Psi(t)=i p \pi \int_{1}^{t}\left(|\hat{w}(\tau)|^{2}-|\hat{w}(t)|^{2}\right)\right) \frac{d \tau}{\tau} .
$$

Then

$$
\Psi(t)-\Psi(s)=i p \pi \int_{s}^{t}\left(|\hat{w}(\tau)|^{2}-|\hat{w}(t)|^{2}\right) \frac{d \tau}{\tau}+i p \pi\left(|\hat{w}(t)|^{2}-|\hat{w}(s)|^{2}\right) \log s,
$$

where $1<s<\tau<t$. Using (4.23), we get

$$
\|\Psi(t)-\Psi(s)\|_{\infty} \leq C \epsilon \int_{s}^{t} \tau^{-1-\gamma+C \epsilon} d \tau+C \epsilon s^{-\gamma+C \epsilon} \log s \leq C \epsilon s^{-\gamma+C \epsilon} .
$$

Therefore by (4.25) we see that there exists a unique function $\Phi \in L^{\infty}, i \Phi=$ $\lim _{t \rightarrow \infty} \Psi(t)$, satisfying

$$
\|i \Phi-\Psi(t)\|_{\infty} \leq C \epsilon t^{-\gamma+C \epsilon} .
$$

By (4.24), (4.26) and the identity

$$
\left.i p \pi \int_{1}^{t}|\hat{w}(\tau)|^{2} \frac{d \tau}{\tau}=i p \pi|W|^{2} \log t+i \Phi+(\Psi(t)-i \Phi)+i p \pi\left(|\hat{w}(t)|^{2}-|W|^{2}\right)\right) \log t
$$

we have estimate (1.6) of Theorem 1.4. And from (1.5) and (1.6) the estimate (1.8) follows. The asymptotic formula (1.7) now follows from the identity (2.4) and estimates (2.5), (1.8). This completes the proof of Theorem 1.4.

\section{ACKNOWLEDGMENTS}

One of the authors (P.I.N.) wishes to express his deep gratitude to Consejo Nacional de Ciencia y Tecnologia de Mexico (Conacyt) for their support. He is also grateful to Instituto de Fisica y Matematica for kind hospitality. The authors would like to thank Professor D. B. Dix for useful comments on Theorem 1.2.

\section{REFERENCES}

1. L.Abdelouhab, Nonlocal dispersive equations in weighted Sobolev spaces, Differential and Integral Equations 5 (1992), 307-338. MR 92k:35226

2. L. Abbelouhab, J. L. Bona, M. Felland and J. C. Saut, Nonlocal models for nonlinear dispersive waves, Phys.D 40 (1989), 360-392. MR 91d:58033

3. A. S. Fokas and M. J. Ablowitz, The inverse scattering transform for the Benjamin-Ono equation - a pivot to multidimensional problems, Stud. Appl. Math. 68(1) (1983), 1-10. MR 84f:35139

4. C. J. Amick and J. F. Toland, Uniqueness and related analytic properties for the BenjaminOno equation - a nonlinear Neumann problem in the plane, Acta Math. 167 (1991), 107-126. MR 92i:35099

5. T. B. Benjamin, Internal waves of permanent form in fluids of great depth, J. Fluid Mech. 29 (1967), 559-592.

6. T. L. Bock and M. D. Kruskal, A two parameter Miura transformation of the Benjamin-Ono equation, Phys. Lett. A 74 (1979), 173-176. MR 82d:35083 
7. K. M. Case, Benjamin-Ono related equations and their solutions, Proc. Nat. Acad. Sci. U.S.A. 76 (1979), 1-3. MR 82c:76106

8. R. R. Coifman and M. V. Wickerhauser, The scattering transform for the Benjamin-Ono equation, Inverse Probl. 6 (1990), 825-861. MR 91j:34136

9. P. Constantin, J. C. Saut, Local smoothing properties of dispersive equations, J. Amer. Math. Soc. 1 (1988), 413-439. MR 89d:35150

10. A. Friedman, Partial Differential Equations, New York, Holt, Rinehart and Winston, 1969. MR 56:3433

11. J. Ginibre and T. Ozawa, Long range scattering for nonlinear Schrödinger and Hartree equations in space dimension $n \geq 2$, Commun. Math. Phys. 151 (1993), 619-664. MR 93m:35168

12. J. Ginibre and G. Velo, Smoothing properties and existence of solutions for the generalized Benjamin-Ono equation, J. Diff. Eqs. 93 (1991), 150-212. MR 93b:35116

13. J. Ginibre and G. Velo, Properties de lissage et existence de solutions pour l'equation de Benjamin-Ono generalisee, C. R. Acad. Sci. Paris Sér. I. Math. 308 (1989), 309-314. MR 90b:35207

14. J. Ginibre and G. Velo, Commutator expansions and smoothing properties of generalized Benjamin-Ono equations, Ann. Inst. H. Poincare, Phys. Theor. 51 (1989), 221-229. MR 90m:35167

15. N.Hayashi, K.Kato and T.Ozawa, Dilation method and smoothing effect of solutions to the Benjamin-Ono equation, Proceedings of Royal Society of Edingburgh A 126 (1996), 273-286. MR 97a:35202

16. N.Hayashi and P.I.Naumkin, Asymptotic behavior in time of solutions to the derivative nonlinear Schrödinger equation, Ann. Inst. H. Poincaré (Physique Theorique) (to appear)

17. N.Hayashi and T.Ozawa, Modified wave operators for the derivative nonlinear Schödinger equations, Math. Annalen 298 (1994), 557-576. MR 95f:35240

18. R.J.Iorio, On the Cauchy problem for the Benjamin-Ono equation, Comm. Partial Differential Equations 11 (1986), 1031-1081; 16 (1991), 531-532. MR 88b:35034; MR 92b:35133

19. R.J.Iorio, The Benjamin-Ono equations in weighted Sobolev spaces, J. Math. Anal. Appl. 157 (1991), 577-590. MR 92d:35251

20. C.E.Kenig, G.Ponce and L.Vega, Oscillatory integrals and regularity of dispersive equations, Indiana Univ. Math. J. 40 (1991), 33-69. MR 92d:35081

21. C.E.Kenig, G.Ponce and L.Vega, On the generalized Benjamin-Ono equation, Trans. Amer. Math. Soc. 342 (1994), 155-172. MR 94e:35121

22. P.I.Naumkin, Asymptotics for large time for nonlinear Schrödinger equation, The Proceedings of the 4th MSJ International Reserch Institute on "Nonlinear Waves", GAKUTO International Series, Mathematical Sciences and Applications, Gakkotosho, 1996 (to appear).

23. P.I.Naumkin, Asymptotics for large time for nonlinear Schrödinger equation (in Russian), Izv. Ross. Akad. Nauk Ser. Mat. 61 (1997), no. 4, 81-118; English transl., to appear in Russian Acad. Sci. Izv. Math. CMP 98:04

24. V.L.Nunes Wagner, On the well-posedness and scattering for the transitional Benjamin-Ono equation, Second Workshop on PDE (Rio de Janeiro, 1991), Mat. Contemp. 3 (1992), 127148.

25. H.Ono, Algebraic solitary waves in stratified fluids, J.Phys. Soc. Japan 39 (1975), 1082-1091. MR 53:2129

26. T.Ozawa, Long range scattering for nonlinear Schrödinger equations in one space dimension, Commun. Math. Phys. 139 (1991), 479-493. MR 92j:35172

27. A.Nakamura, Backlund transformations and conservation laws of the Benjamin-Ono equation, J.Phys.Soc.Japan 47(4) (1979), 1335-1340. MR 80m:35068

28. G.Ponce, Regularity of solutions to nonlinear dispersive equations, J.Diff.Eq. 78 (1989), 122135. MR 90c: 35031

29. G.Ponce, Smoothing properties of solutions of the Benjamin-Ono equation, Analysis and Partial Differential Equations, Lecture Notes Pure Appl. Math., vol. 122, Marcel Dekker, 1990, pp. 667-679. MR 91c:35135

30. G.Ponce, On the global well-posedness of the Benjamin-Ono equation, Differential and Integral Equations 4 (1991), 527-542. MR 92e:35137

31. J.C.Saut, Sur quelques generalisations de l'equation de Korteweg-de Vries, J. Math. Pures Appl. 58 (1979), 21-61. MR 82m:35133

32. P.M.Santini, M.J.Ablowitz, A.S.Fokas, On the limit from the intermediate long wave equation to the Benjamin-Ono equation, J. Math. Phys. 25 (1984), 892-899. MR 85k:35214 
33. E.M.Stein, Singular Integrals and Differentiability Properties of Functions, Princeton Univ. Press, Princeton Math. Series 30, 1970. MR 44:7280

34. M.Tanaka, Nonlinear self-modulation problem of the Benjamin-Ono equation, Phys.Soc.Japan 51 (1982), 2686-2692. MR 83k:76081

35. M.M.Tom, Smoothing properties of some weak solutions of the Benjamin-Ono equation, Differential and Integral Equations 3 (1990), 683-694. MR 91e:35191

Department of Applied Mathematics, Science University of Tokyo, 1-3, Kagurazaka, SHINJUKU-KU, TOKYO 162, JAPAN

E-mail address: nhayashi@rs.kagu.sut.ac.jp

Instituto de Fisica y Matematica, Universidad Michoacana, AP 2-82, CP 58040, Morelia, Michoacana, Mexico

E-mail address: naumkin@ifm1.ifm.umich.mx 